\title{
Grass pollen nasal challenge is associated with increases in Th2 cytokines, Eotaxin, MDC and IL-6 in nasal fluid
}

\author{
Guy Scadding ${ }^{1 *}$, Aarif Eifan², Martin Penagos ${ }^{2}$, Gitte Konsgaard Koed ${ }^{3}$, Peter Adler Wurtzen ${ }^{3}$, Mohamed Shamji $^{2}$, \\ Stephen Durham²
}

From 9th Symposium of Experimental Rhinology and Immunology of the Nose (SERIN 2013)

Leuven, Belgium. 21-23 March 2013

\section{Background}

We previously validated a grass pollen nasal challenge model to record clinical outcomes and local biomarkers in nasal fluid [1]. Here we further validate our methods and compare the expression of Th2- and Th1-related cytokines, chemokines and IL-17.

\section{Methods}

18 grass pollen allergics underwent nasal allergen challenges; 15 grass allergics had matched diluent challenges. Symptom scores and peak nasal inspiratory flow were recorded; nasal fluid was collected using polyurethane sponges, then extracted by centrifugation across microfilters. Fluid was then tested for Th2 cytokines by Mesoscale 7-plex multi-spot plate assay. A further 6 allergics underwent nasal allergen challenges with collection of fluid using both polyurethane sponges and Leukosorb filter strips; nasal fluid was analysed by Milliplex cytokine/chemokine magnetic bead multiplex assay.

\section{Results}

Allergen vs diluent challenges; multi-spot plate assay: symptoms increased, and peak nasal flow decreased, following allergen but not diluent challenge (both $\mathrm{p}<0.001$, between groups difference). Levels of IL-4 ( $<<0.01)$, IL-5 and IL-13 (both $\mathrm{P}<0.001$ ) were maximally increased at 5 hours compared to pre-challenge; no significant increases were seen following diluent challenge. Between group differences (allergen vs diluent) for IL-4, -5 and -13 were seen at 4 and 6 hours (all $\mathrm{p}<0.01$ ).Allergen challenge; magnetic bead assay: IL-5 was increased at

Imperial College, London, UK

Full list of author information is available at the end of the article
6 hours ( $\mathrm{p}=0.03$ vs pre-challenge), with IL-13 and IL-4 also showing a trend towards an increase (both $\mathrm{p}=0.06$ vs pre-challenge). Eotaxin and MDC were increased at 6 hours (both $\mathrm{p}=0.03$ vs pre-challenge); IL- 6 was elevated at 2 hours ( $p=0.03$ vs pre-challenge). Levels of IL17A, IL-27, IL-23, IFN-gamma and IL-12p70 were low and did not change significantly after allergen challenge. High levels of IL-8 were detected, maximal at baseline, but did not change significantly after challenge. Polyurethane sponges proved superior to filters for all measurements.

\section{Conclusions}

Grass pollen nasal challenge is associated with a strong local Th2, but not Th1, response detectable in nasal secretions. Despite elevation of IL-6, there is no significant local Th17 response, up to 6 hours. Responses are allergendriven and independent of the intervention and any diurnal variation. Multiplex assays are capable of detecting cytokines and chemokines in low volumes $(25 \mathrm{mcl})$ of nasal fluid by both multi-spot and micro-bead techniques.

\section{Author details \\ ${ }^{1}$ Imperial College, London, UK. ${ }^{2}$ Imperial College, London, Allergy and Clinical Immunology, London, UK. ${ }^{3}$ ALK-Abello, Vaccine Research and Discovery, Hørsholm, Denmark.}

Published: 16 July 2013

\section{Reference}

1. J Immunol Methods 2012, 384(1-2):25-32.

doi:10.1186/2045-7022-3-S2-P29

Cite this article as: Scadding et al:: Grass pollen nasal challenge is associated with increases in Th2 cytokines, Eotaxin, MDC and IL-6 in nasal fluid. Clinical and Translational Allergy 2013 3(Suppl 2):P29.

\section{C)

(C) 2013 Scadding et al; licensee BioMed Central Ltd. This is an Open Access article distributed under the terms of the Creative Commons Attribution License (http://creativecommons.org/licenses/by/2.0), which permits unrestricted use, distribution, and reproduction in any medium, provided the original work is properly cited. 